TITLE:

\title{
Structural stability and electronic properties of SiC nanocones: First- principles calculations and symmetry considerations
}

$\operatorname{AUTHOR(S):~}$

Alfieri, G.; Kimoto, T.

\section{CITATION:}

Alfieri, G....[et al]. Structural stability and electronic properties of SiC nanocones: Firstprinciples calculations and symmetry considerations. APPLIED PHYSICS LETTERS 2011, 98(12): 123102.

\section{ISSUE DATE:}

2011-03-21

URL:

http://hdl.handle.net/2433/160626

\section{RIGHT:}

Copyright 2011 American Institute of Physics. This article may be downloaded for personal use only. Any other use requires prior permission of the author and the American Institute of Physics. The following article appeared in APPLIED PHYSICS LETTERS 98, 123102 (2011) and may be found at http://link.aip.org/link/?apl/98/123102 


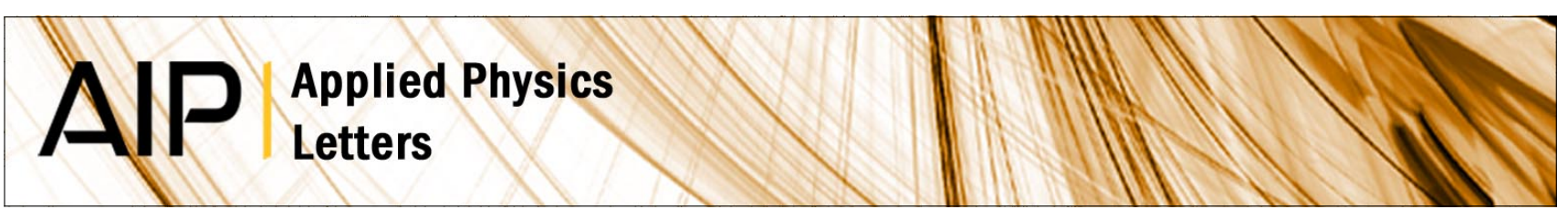

\section{Structural stability and electronic properties of SiC nanocones: First- principles calculations and symmetry considerations}

G. Alfieri and T. Kimoto

Citation: Appl. Phys. Lett. 98, 123102 (2011); doi: 10.1063/1.3567535

View online: http://dx.doi.org/10.1063/1.3567535

View Table of Contents: http://apl.aip.org/resource/1/APPLAB/v98/i12

Published by the American Institute of Physics.

\section{Related Articles}

Development of a new laser heating system for thin film growth by chemical vapor deposition Rev. Sci. Instrum. 83, 094701 (2012)

Hard x-ray photoemission spectroscopy of Bi2S3 thin films J. Appl. Phys. 112, 053705 (2012)

The role of structural properties on deep defect states in Cu2ZnSnS4 studied by photoluminescence spectroscopy

Appl. Phys. Lett. 101, 102102 (2012)

Hydrostatic pressure and strain effects in short period $\operatorname{lnN} / \mathrm{GaN}$ superlattices

Appl. Phys. Lett. 101, 092104 (2012)

Structural and optoelectronic properties, and infrared spectrum of cubic $\mathrm{BaSnO} 3$ from first principles calculations J. Appl. Phys. 112, 043703 (2012)

\section{Additional information on Appl. Phys. Lett.}

Journal Homepage: http://apl.aip.org/

Journal Information: http://apl.aip.org/about/about_the_journal

Top downloads: http://apl.aip.org/features/most_downloaded

Information for Authors: http://apl.aip.org/authors

\section{ADVERTISEMENT}

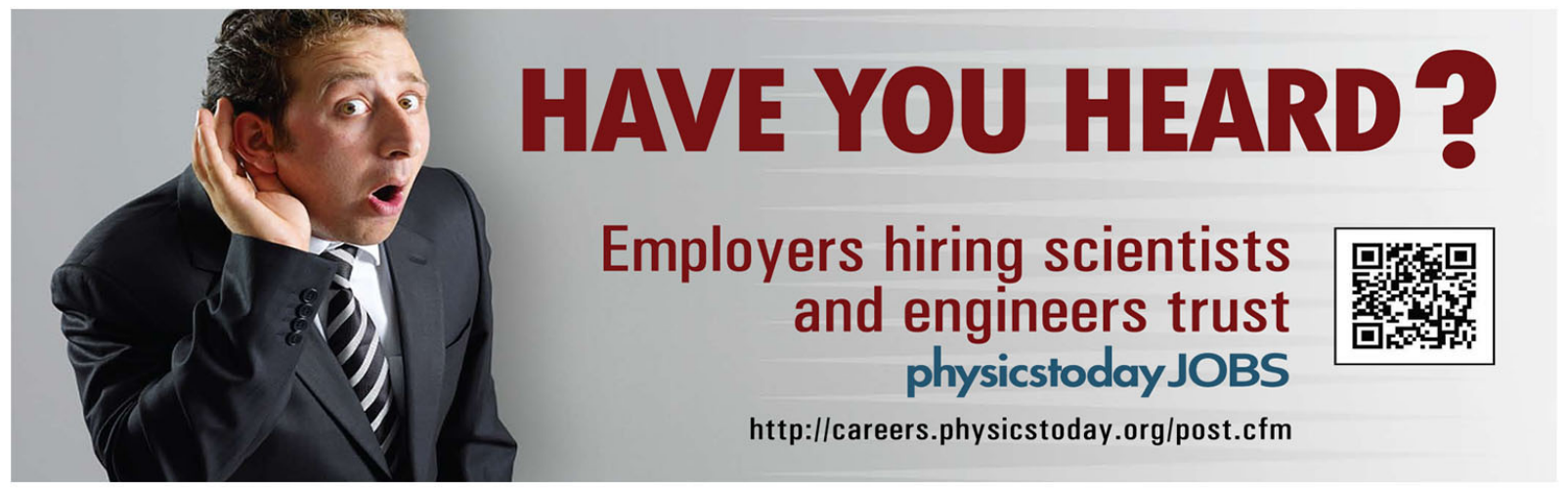




\title{
Structural stability and electronic properties of SiC nanocones: First-principles calculations and symmetry considerations
}

\author{
G. Alfieri ${ }^{1, a)}$ and T. Kimoto ${ }^{1,2}$ \\ ${ }^{1}$ Department of Electronic Science and Engineering, Kyoto University, Kyotodaigaku-katsura, Nishikyo, \\ Kyoto 615-8510, Japan \\ ${ }^{2}$ Photonics and Electronics Science and Engineering Center, Kyoto University, Kyotodaigaku-katsura, \\ Nishikyo, Kyoto 615-8510, Japan
}

(Received 4 February 2011; accepted 25 February 2011; published online 21 March 2011)

\begin{abstract}
The structural and electronic properties of $\mathrm{SiC}$ nanocones ( $\mathrm{SiCNCs}$ ), as a function of the disclination angle and electric field intensity and orientation, are investigated by means of ab initio calculations. Phonon analysis revealed that SiCNCs with only three disclination angles are allowed and, among these, the band gap of only one SiCNC is affected by a transverse electric field. An interpretation of these findings, from a group theoretical point of view, is also given. (C) 2011 American Institute of Physics. [doi:10.1063/1.3567535]
\end{abstract}

Nanomaterials can find a great number of applications in modern-day technology, ranging from household to on-board satellite electronics, and because of the increasing need for speed and miniaturization of electronic devices, nanotechnology has become a very active field of study. At the same time, due the rapid development silicon carbide $(\mathrm{SiC})$ technology, the existence of $\mathrm{SiC}$ nanostructures that can exploit the superior physical- chemical properties of SiC (Ref. 1) has been investigated and, in the last few years, many studies on $\mathrm{SiC}$ nanotubes have been reported in the literature. ${ }^{2-4} \mathrm{On}$ the contrary, little information on $\mathrm{SiC}$ nanocones ( $\mathrm{SiCNCs}$ ) is available. ${ }^{5}$ This is unfortunate, because it was proven that nanocones arrays for solar panels applications can be beneficial in terms of increased optical absorption coefficient ${ }^{6}$ and since space probes rely on photovoltaic power generation, solar panels based on SiCNCs arrays can be a valid alternative in terms of high-temperature, high-light intensity and high radiation conditions.

In this study, we investigated the structural and electronic properties of $\mathrm{SiCNCs}$, obtained by rolling a single $\mathrm{SiC}$ layer with $\mathrm{Si}-\mathrm{C}$ bond length of $1.78 \AA$, as a function of the disclination angle $d$ (defined as the angle of the sector removed from a flat $\mathrm{SiC}$ sheet to form a cone), ranging from $60^{\circ}$ to $300^{\circ}$, and of an electric field $F$ (parallel or perpendicular to the cone axis), of intensities ranging from 0.05 to $0.20 \mathrm{~V} / \AA ̊$. For this purpose, we employed the $a b$ initio calculation code SIESTA (Ref. 7) using a double- $\zeta$ plus polarization atomic orbitals basis set (a 0.02 Ry energy shift to compress the basis set was used to speed up calculations) and by taking the effect of core electrons into account by TroullierMartins norm-conserving pseudopontials. ${ }^{8}$ The PerdewZunger form ${ }^{9}$ of the local-density approximation (LDA) was used for the exchange-correlation potential. The equilibrium configuration of the investigated SiCNCs (with hydrogen passivated dangling bonds) was obtained by relaxing the atomic coordinates with a conjungate gradient algorithm until the maximum atomic forces were less than $0.020 \mathrm{~V} / \AA$ and the stress tensor less than $0.5 \mathrm{GPa}$. The charge density was projected onto a real space grid with an equivalent cutoff of 70 Ry ensured the convergence of the $\Gamma$-point calculations.

${ }^{\text {a)} E l e c t r o n i c ~ m a i l: ~ g i o v a n n i @ s e m i c o n . k u e e . k y o t o-u . a c . j p . ~}$
We also employed the Perdew-Burke-Ernzerhof (PBE) (Ref. 10) form of the generalized gradient approximation (GGA) for the exchange-correlation potential and the calculated band gap width, $E_{\mathrm{GAP}}$, and spontaneous dipole moment, $D$, are also shown in parenthesis in Table I, for comparison.

The stoichiometry of the investigated SiCNCs is reported in Table I and, it can be seen that, except for $d$ $=120^{\circ}$ and $240^{\circ}$, they can be classified into C-rich or Si-rich. It can be noted that $\mathrm{C}$-rich $\mathrm{SiCNCs}$ possess a wider $E_{\mathrm{GAP}}$ than Si-rich SiCNCs and also wider $d$ corresponds to more narrow $E_{\mathrm{GAP}}$, because of increased curvature effect. ${ }^{2}$ The symmetry of all the investigated SiCNCs (third column of Table I) is $C_{s}$ with the exception of $d=120^{\circ}, C_{2 v}$, which, interestingly, corresponds to the highest $E_{\mathrm{GAP}}$ value. Knowing the symmetry of the investigated SiCNCs is particularly useful because, being $C_{s}$ or $C_{2 v}$, this implies the presence of a spontaneous $\mathbf{D}$ which, as it can be seen in the sixth column of Table I, is indeed the case ( $\mathbf{D}$ is parallel to the cone axis or slightly deviating from it). In the last column of Table I, the binding energy per atom, $E_{B}$, as defined in Ref. 4 shows no correlation to either $d$ or point group symmetry, and the values are $1-2 \mathrm{eV}$ larger than those reported by Mavrandonakis et $a l^{5}$ in nanotube-based conical nanostructures, possibly due to the different calculation method employed.

In order to check the structural stability of the SiCNCs of the present study (or, equivalently, that the relaxed geometry is preserved), we analyzed the phonon modes (at the

TABLE I. Stoichiometry, disclination angle, point group symmetry, band gap energy, dipole, and binding energy for the eight investigated SiCNCs. In parenthesis the value of the band gap and dipole calculated by GGA-PBE.

\begin{tabular}{lcccccc}
\hline \hline Stoichiometry & $\begin{array}{c}d \\
(\mathrm{deg})\end{array}$ & $\begin{array}{c}\text { Point } \\
\text { group }\end{array}$ & $\begin{array}{c}\text { Tip point } \\
\text { group }\end{array}$ & $\begin{array}{c}E_{\mathrm{GAP}} \\
(\mathrm{eV})\end{array}$ & $D(\mathrm{D})$ & $\begin{array}{c}E_{B} \\
\text { (eV/atom) }\end{array}$ \\
\hline $\mathrm{C}_{36} \mathrm{Si}_{34} \mathrm{H}_{20}$ & 60 & $C_{s}$ & $C_{s}$ & $0.86(0.87)$ & $2.17(2.6)$ & 6.83 \\
$\mathrm{C}_{34} \mathrm{Si}_{36} \mathrm{H}_{20}$ & 60 & $C_{s}$ & $C_{2 v}$ & $0.59(0.58)$ & $2.35(2.5)$ & 6.73 \\
$\mathrm{C}_{32} \mathrm{Si}_{32} \mathrm{H}_{16}$ & 120 & $C_{2 v}$ & $C_{2 v}$ & $1.42(1.38)$ & $3.29(2.98)$ & 6.85 \\
$\mathrm{C}_{36} \mathrm{Si}_{33} \mathrm{H}_{15}$ & 180 & $C_{s}$ & $C_{2 v}$ & $0.58(0.58)$ & $7.7(7.4)$ & 6.95 \\
$\mathrm{C}_{33} \mathrm{Si}_{36} \mathrm{H}_{15}$ & 180 & $C_{s}$ & $C_{2 h}$ & $0.50(0.51)$ & $14.4(14.0)$ & 6.81 \\
$\mathrm{C}_{46} \mathrm{Si}_{46} \mathrm{H}_{16}$ & 240 & $C_{s}$ & $C_{\infty v}$ & $0.31(0.30)$ & $16.3(16.1)$ & 7.09 \\
$\mathrm{C}_{46} \mathrm{Si}_{38} \mathrm{H}_{10}$ & 300 & $C_{s}$ & $D_{3 h}$ & $0.29(0.29)$ & $14.1(6.85)$ & 7.28 \\
$\mathrm{C}_{38} \mathrm{Si}_{46} \mathrm{H}_{10}$ & 300 & $C_{s}$ & $D_{3 d}$ & $0.08(0.08)$ & $6.2(6.4)$ & 6.72 \\
\hline \hline
\end{tabular}




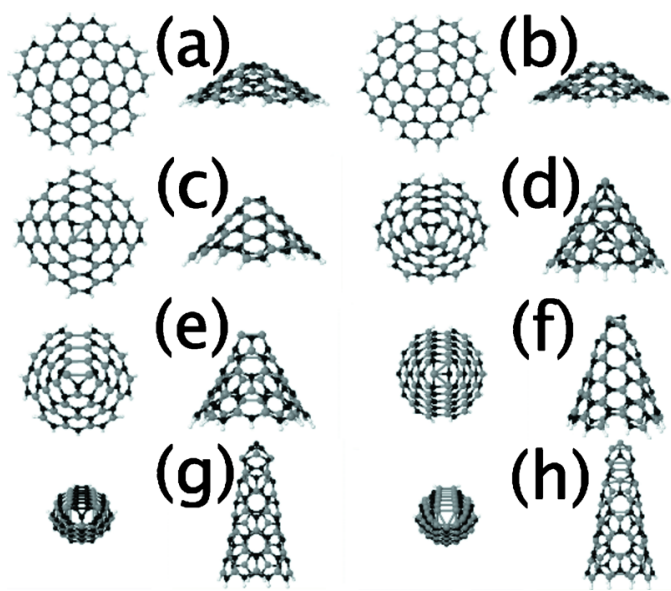

FIG. 1. (Color online) Top and side view of SiCNCs with disclination angles of (a) $60^{\circ}$ (C-rich), (b) $60^{\circ}$ (Si-rich), (c) $120^{\circ}$, (d) $180^{\circ}$ (C-rich), (e) $180^{\circ}$ (Si-rich), (f) $240^{\circ}$, (g) $300^{\circ}$ (C-rich), and (h) $300^{\circ}$ (Si-rich). C-atoms are black, Si-atoms gray, and $\mathrm{H}$-atoms white.

$\Gamma$-point) calculated with force constant method. ${ }^{7}$ This analysis provides a reliable stability test, as the presence of imaginary phonon frequencies suggests that the system is structurally unstable and a structural transformation can occur. Our results indicate the presence of imaginary frequencies in all the investigated SiCNCs, with the exception of the $\mathrm{C}_{36} \mathrm{Si}_{34} \mathrm{H}_{20}\left[d=60^{\circ}, C_{s}\right.$, Fig. 1(a) $], \mathrm{C}_{32} \mathrm{Si}_{32} \mathrm{H}_{16}\left[d=120^{\circ}, C_{2 v}\right.$, Fig. 1(c)], and $\mathrm{C}_{46} \mathrm{Si}_{38} \mathrm{H}_{10}\left[d=300^{\circ}, C_{s}\right.$, Fig. $\left.1(\mathrm{~g})\right]$. We point out that the three stable SiCNCs are either $\mathrm{C}$-rich or possess a ratio of $\mathrm{C} / \mathrm{Si}=1$, in agreement with the literature. ${ }^{11}$ Yet, this is not sufficient to justify their stability, as the other $\mathrm{C}$-rich or $\mathrm{C} / \mathrm{Si}=1 \mathrm{SiCNCs}$ display imaginary phonon frequencies.

Charlier and Rignanese ${ }^{12}$ suggested that the presence of particular topological defects at the tip can contribute to the stability of nanocones. We take advantage of this finding to analyze the tip of our SiCNCs and propose a criterion for determining their stability. As it can be seen in Fig. 1, the tip of studied SiCNCs is penthagonal $\left[d=60^{\circ}\right.$, Figs. 1(a) and 1(b)], rhombohedral $\left[d=120^{\circ}\right.$, Fig. 1(c) ], triangular (scalene/ isosceles) $\left[d=180^{\circ}\right.$, Figs. $1(\mathrm{~d})$ and $\left.1(\mathrm{e})\right]$, linear $\left[d=240^{\circ}\right.$, Figs. 1(f) $]$, and triangular (equilateral/scalene) $\left[d=300^{\circ}\right.$, Figs. $1(\mathrm{~g})$ and $1(\mathrm{~h})$ ]. The SiCNCs' tips point group symmetries, are reported in Table I (column 4). A general rule can be deduced, as the point group of unstable SiCNC is different from that of the tip, while for stable SiCNCs the total point group and that of the tip coincide (in the case of $\mathrm{C}_{46} \mathrm{Si}_{38} \mathrm{H}_{10}, C_{s}$ is found in the direct product expression of $D_{3 h}=D_{3} \otimes C_{s}$ while for $\mathrm{C}_{38} \mathrm{Si}_{46} \mathrm{H}_{10}, D_{3 d}=D_{3} \otimes C_{i}$ ).

Next, we analyze the effects of $\mathbf{F}_{\perp}$ and $\mathbf{F}_{\|}$, the electric field perpendicular and parallel to the cone axis, respectively, on $E_{B}$ and $E_{\mathrm{GAP}}$ for the three stable SiCNCs. No effect of the intensity and orientation of $\mathbf{F}$ on $E_{B}$ could be observed for neither of the three SiCNCs. Analogously, negligible effects on the $E_{\mathrm{GAP}}$ can be observed for the $d=60^{\circ} \mathrm{SiCNC}$ $\left(\mathrm{C}_{36} \mathrm{Si}_{34} \mathrm{H}_{20}\right)$, with a 0.03 and $0.01 \mathrm{eV}$ decrease in $E_{\mathrm{GAP}}$ after application of either $\mathbf{F}_{\perp}$ or $\mathbf{F}_{\|}$, respectively, of intensities of $0.20 \mathrm{~V} / \AA$. The same applies to the $300^{\circ} \mathrm{SiCNC}\left(\mathrm{C}_{46} \mathrm{Si}_{38} \mathrm{H}_{10}\right)$ (0.01 eV and $0.02 \mathrm{eV}$ for $\mathbf{F}_{\perp}$ or $\mathbf{F}_{\|}$, respectively. On the contrary a stronger decrease in $E_{\mathrm{GAP}}$ is observed for the $\mathrm{SiCNC}$ of $d=120^{\circ}\left(\mathrm{C}_{32} \mathrm{Si}_{32} \mathrm{H}_{16}\right)$. Application of $\mathbf{F}_{\perp}$, of intensities $0.05,0.10,0.15$, and $0.20 \mathrm{~V} / \AA$, results in a decrease in

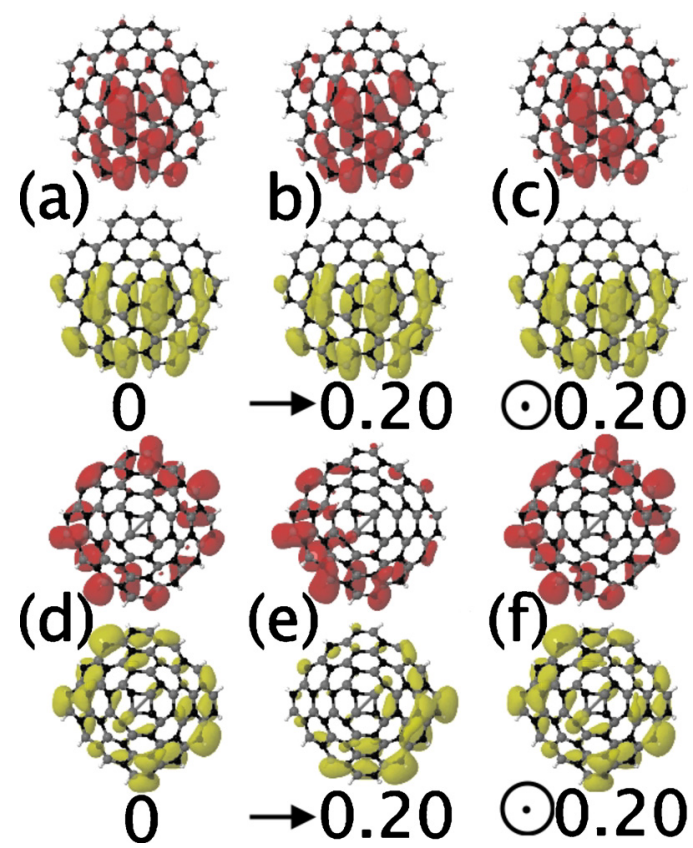

FIG. 2. (Color online) Behavior of the lowest unoccupied (first row) and highest occupied (second row) molecular orbitals for $\mathrm{C}_{36} \mathrm{Si}_{34} \mathrm{H}_{20}$ under (a) no, (b) transverse, and (c) parallel, to the cone axis, $0.20 \mathrm{~V} / \AA$ electric field. The same is shown for $\mathrm{C}_{32} \mathrm{Si}_{32} \mathrm{H}_{16}[(\mathrm{~d})$, (e), and (f)].

$E_{\mathrm{GAP}}$ of $1.35,1.20,1.05$, and $0.89 \mathrm{eV}$, respectively. By changing the orientation of the electric field, that means by applying $\mathbf{F}_{\|}, E_{\mathrm{GAP}}$ becomes less sensitive to the field $\left(E_{\mathrm{GAP}}\right.$ changes as $1.41 \mathrm{eV}, 1.41 \mathrm{eV}, 1.40 \mathrm{eV}$, and $1.40 \mathrm{eV}$ for 0.05 $\mathrm{V} / \AA ̊$, $0.10 \mathrm{~V} / \AA ̊ ., 0.15 \mathrm{~V} / \AA$, and $0.20 \mathrm{~V} / \AA$, respectively).

In Fig. 2, the behavior of the lowest unoccupied and highest occupied molecular orbital, LUMO (red) and HOMO (yellow), respectively, is shown for the $\mathrm{C}_{36} \mathrm{Si}_{34} \mathrm{H}_{20}$ and $\mathrm{C}_{32} \mathrm{Si}_{32} \mathrm{H}_{16} \mathrm{SiCNCs}$. It can be seen that application of either $\mathbf{F}_{\perp}$ [Fig. 2(b)] or $\mathbf{F}_{\|}$[Fig. 2(c)], with intensities of $0.20 \mathrm{~V} / \AA$, on the $\mathrm{C}_{36} \mathrm{Si}_{34} \mathrm{H}_{20}$ cone yields no effect on the molecular orbitals and, in fact, no change is observed on the $E_{\mathrm{GAP}}$ values. For the case of $\mathrm{C}_{32} \mathrm{Si}_{32} \mathrm{H}_{16}, F_{\perp}=0.20 \mathrm{~V} / \AA$ results in the rearrangement of the HOMO (negative charges) and LUMO (positive charges) along the direction of field, at two opposite sides of the SiCNC. This means that two regions are formed, one of low (HOMO) and one of high (LUMO) electrostatic potential that, in terms of energy, contribute to the lowering of the LUMO and the rising of the HOMO, thus shrinking the $E_{\mathrm{GAP}}$. When $\mathbf{F}_{\|}$is applied, no rearrangement of either HOMO or LUMO can be noted and $E_{\mathrm{GAP}}$ does not change. As the SiCNC with $C_{2 v}$ symmetry is more responsive to $\mathbf{F}_{\perp}$ than those with $C_{s}$ symmetry it is straightforward asking whether or not this can be interpreted by using group theory. To do this, for simplicity, we consider only the HOMO, namely the C-atoms of the $\mathrm{C}_{36} \mathrm{Si}_{34} \mathrm{H}_{20}\left(C_{s}\right)$ and $\mathrm{C}_{32} \mathrm{Si}_{32} \mathrm{H}_{16}\left(\mathrm{C}_{2 \mathrm{v}}\right)$ SiCNCs.

By employing the directed valence (DV) bonding ${ }^{13}$ on $\mathrm{C}$-atoms, we obtain the following irreducible representations (irrep) $\Gamma_{C_{s}}^{\text {irrep }}=19 A^{\prime}+17 A^{\prime \prime} \quad\left(\mathrm{C}_{36} \mathrm{Si}_{34} \mathrm{H}_{20}\right) \quad$ and $\Gamma_{C_{2 v}}^{\text {irrep }}=10 A_{1}$ $+6 A_{2}+8 B_{1}+8 B_{2}\left(\mathrm{C}_{32} \mathrm{Si}_{32} \mathrm{H}_{16}\right)$. By direct product multiplication, with the representations of the $s, p_{x}$, and $p_{y}$ atomic orbitals of the respective point-symmetry groups $\left(A^{\prime}, A^{\prime \prime}\right.$ and $A_{1}, B_{1}, B_{2}$ for $C_{s}$ and $C_{2 v}$, respectively) we obtain 


$$
\begin{aligned}
\Gamma_{C_{s}}^{\mathrm{DV}}= & \underbrace{\left(19 A^{\prime \prime}+17 A^{\prime}\right)}_{s}+\underbrace{\left(19 A^{\prime}+17 A^{\prime \prime}\right)}_{p_{x}, p_{y}}, \\
\Gamma_{C_{2 v}}^{\mathrm{DV}}= & \underbrace{\left(10 A_{1}+6 A_{2}+8 B_{1}+8 B_{2}\right)}_{s} \\
& +\underbrace{\left(10 B_{1}+6 B_{2}+8 A_{1}+8 A_{2}\right)}_{p_{x}} \\
& +\underbrace{\left(10 B_{2}+6 B_{1}+8 A_{2}+8 A_{1}\right)}_{p_{y}},
\end{aligned}
$$

meaning that the orbitals involved are $s, p_{x}$, and $p_{y}$, corresponding to the $s p^{2}$ hybridization of the single $\mathrm{SiC}$ sheet. In order to evaluate the effects of $\mathbf{F}$ on the SiCNCs, the $\Gamma^{\mathrm{DV}}$ 's are multiplied, by direct product multiplication, with the representations of $\mathbf{F}_{\perp}$ and $\mathbf{F}_{\|}$of the respective point-symmetry groups that are $A^{\prime}, A^{\prime \prime}\left(C_{s}\right)$ and $B_{1}, A_{1}\left(C_{2 v}\right)$, for $\mathbf{F}_{\perp}$ and $\mathbf{F}_{\|}$, respectively. For $C_{s}, \Gamma_{C_{s}}^{D V} \otimes A^{\prime}$ and $\Gamma_{C_{s}}^{D V} \otimes A^{\prime \prime}$ still result in $\Gamma_{C_{s}}^{\mathrm{DV}}$. The same is obtained for $C_{2 v}$ as $\Gamma_{C_{2 v}}^{\mathrm{DV}} \otimes A_{1}=\Gamma_{C_{2 v}}^{\mathrm{DV}}$, however,

$$
\begin{aligned}
\Gamma_{C_{2 v}}^{\mathrm{DV}} \otimes B_{1}= & \underbrace{\left(10 A_{1}+6 A_{2}+8 B_{1}+8 B_{2}\right)}_{s} \\
& +\underbrace{\left(10 B_{1}+6 B_{2}+8 A_{1}+8 A_{2}\right)}_{p_{\mathrm{x}}} \\
& +\left(10 A_{2}+6 A_{1}+8 B_{2}+8 B_{1}\right) .
\end{aligned}
$$

This suggests that the application of a $\mathbf{F}_{\perp}$ to a SiCNC with $C_{2 v}$ symmetry results in the coupling of $\Gamma_{C_{2 v} v}^{\mathrm{DV}}$ with a final state containing the term $\left(10 A_{2}+6 A_{1}+8 B_{2}+8 B_{1}\right)$ not present in the initial. On the contrary, no change in the initial and final states is observed when either $\mathbf{F}_{\perp}$ or $\mathbf{F}_{\|}$is applied to a SiCNC of $C_{s}$ symmetry, suggesting that the response to an electric field is different from that of SiCNs with $C_{2 v}$ symmetry (nil or slower response).

In conclusion, phonon analysis revealed that $\mathrm{SiCNCs}$ with $d=60^{\circ}, 120^{\circ}$, and $300^{\circ}$ and $\mathrm{C} / \mathrm{Si} \geq 1$ are stable and only the $E_{\mathrm{GAP}}$ of the $\mathrm{SiCNC}$ with $d=120^{\circ}$, is affected by a transverse $\mathbf{F}$. These findings were interpreted by means of group theory, by considering the correspondence between the point group symmetry of the cone with that of the shape of the tip and in terms of symmetry change in the irrep of the HOMO, when an electric field is applied.

This work was supported by the Japan Society for the Promotion of Science and by the Global COE Program (C09) from the Ministry of Education, Culture, Sports, and Technology, Japan.

${ }^{1}$ H. Matsunami, Jpn. J. Appl. Phys., Part 1 43, 6835 (2004).

${ }^{2}$ J. J. Wu and G. Y. Guo, Phys. Rev. B 76, 035343 (2007).

${ }^{3}$ A. Gali, Phys. Rev. B 73, 245415 (2006).

${ }^{4}$ G. Alfieri and T. Kimoto, Nanotechnology 20, 285703 (2009).

${ }^{5}$ A. Mavrandonakis, G. E. Froudakis, A. Andriotis, and M. Menon, Appl. Phys. Lett. 89, 123126 (2006).

${ }^{6}$ J. Zhu, Z. Yu, G. F. Burkhard, C. M. Hsu, S. T. Connor, Y. Xu, Q. Wang, M. McGehee, S. Fan, and Y. Cui, Nano Lett. 9, 279 (2009).

${ }^{7}$ J. M. Soler, J. D. Gale, A. Garcia, J. Junquera, P. Ordejon, and D. Sanchez-Portal, J. Phys.: Condens. Matter 14, 2745 (2002).

${ }^{8}$ N. Troullier and J. L. Martins, Phys. Rev. B 43, 1993 (1991)

${ }^{9}$ J. P. Perdew and A. Zunger, Phys. Rev. B 23, 5048 (1981).

${ }^{10}$ J. P. Perdew, K. Burke, and M. Ernzerhof, Phys. Rev. Lett. 77, 3865 (1996).

${ }^{11}$ A. Mavrandonakis, G. E. Froudakis, M. Schnell, and M. Muhlhäuser, Nano Lett. 3, 1481 (2003).

${ }^{12}$ J.-C. Charlier and G.-M. Rignanese, Phys. Rev. Lett. 86, 5970 (2001).

${ }^{13}$ M. S. Dresselhaus, G. Dresselhaus, and A. Jorio, Group Theory (Springer, Berlin, 2008). 